\title{
GPX2 Gene
}

National Cancer Institute

\section{Source}

National Cancer Institute. GPX2 Gene. NCI Thesaurus. Code C104434.

This gene is involved in digestion. 